\section{Detección precoz del cáncer cervicouterino en Chile: tiempo para el cambio}

\author{
JAVIERA LÉNIZ MARTELLI ${ }^{1}$, VANESSA VAN DE WYNGARD ${ }^{2}$, \\ MARCELA LAGOS ${ }^{3}$, MARÍA ISABEL BARRIGA ${ }^{4}$, \\ KLAUS PUSCHEL ILLANES ${ }^{1}$, CATTERINA FERRECCIO READI ${ }^{2,5}$
}

\section{Early detection of cervical cancer in Chile: time for change}

Mortality rates for cervical cancer (CC) in Chile are higher than those of developed countries and it has an unequal socioeconomic distribution. The recognition of human papilloma virus (HPV) as the causal agent of cervical cancer in the early 80's changed the prevention paradigms. Current goals are to prevent $H P V$ infection by vaccination before the onset of sexual activity and to detect $H P V$ infection in women older than 30 years. This article reviews CC prevention and early detection methods, discusses relevant evidence to support a change in Chile and presents an innovation proposal. A strategy of primary screening based on HPV detection followed by triage of HPV-positive women by colposcopy in primary care or by cytological or molecular reflex testing is proposed. Due to the existence in Chile of a well-organized nationwide CC prevention program, the replacement of a low-sensitivity screening test such as the Papanicolau test with a highly sensitive one such as HPV detection, could quickly improve the effectiveness of the program. The program also has a network of personnel qualified to conduct naked-eye inspections of the cervix, who could easily be trained to perform triage colposcopy. The incorporation of new prevention strategies could reduce the deaths of Chilean women and correct inequities.

(Rev Med Chile 2014; 142: 1047-1055)

Key words: Delivery of health care; Early detection of cancer; Human papiloma virus; Uterine cervical neoplasms.
'Departamento de Medicina Familiar, Facultad de Medicina, Pontificia Universidad Católica de Chile. Santiago, Chile.

2Departamento de de Salud Pública, Facultad de Medicina, Pontificia Universidad Católica de Chile. Santiago, Chile.

${ }^{3}$ Departamento de Laboratorios Clínicos, Facultad de Medicina, Pontificia Universidad Católica de Chile. Santiago, Chile.

${ }^{4}$ Departamento de GinecologíaObstetricia, Facultad de Medicina, Pontificia Universidad Católica de Chile. Santiago, Chile. ${ }^{5}$ Advanced Center for Chronic Diseases (ACCDiS), Chile.

Fuentes de apoyo y financiamiento: Este artículo se realizó dentro del marco del estudio FONDECYT № 1090597

Recibido el 14 de agosto de 2013, aceptado el 24 de junio de 2014.

Correspondencia: Javiera Léniz M. Av. Vicuña Mackenna 4686. Macul.

Teléfono: (2)3548688

lenizjaviera@gmail.com
E 1 cáncer cervicouterino (CC) continúa siendo una causa importante de mortalidad en el mundo, especialmente en países en desarrollo, donde la mortalidad por este cáncer es más del doble que la de países desarrollados ${ }^{1}$. Desde el reconocimiento del virus papiloma humano (VPH) como causa necesaria para el desarrollo del $\mathrm{CC}^{2}$, ha cambiado el paradigma de prevención de este cáncer. Ahora esta enfermedad puede evitarse mediante la vacunación de niñas antes del inicio de actividad sexual, en tanto que, en las mujeres que ya iniciaron su vida sexual, la identificación del virus o sus productos está en el centro de las estrategias de detección precoz de lesiones precancerosas. Este artículo revisa la situación del CC en Chile a la luz de estas nuevas estrategias de prevención y de la evidencia nacional e internacional, para proponer algunas innovaciones en la estrategia nacional de prevención.

\section{Cáncer cervicouterino en Chile}

Actualmente en Chile, el CC es la tercera causa de muerte en mujeres entre 20 y 44 años. El año 2010 hubo 578 muertes por CC en el país, 33\% en 
mujeres menores de 55 años ${ }^{3}$. Aunque la mortalidad por CC ha disminuido en la última década, no se logró una reducción de $40 \%$ propuesta por el Ministerio de Salud (MINSAL) para el decenio 2000-20104.

La tasa ajustada de mortalidad en Chile fue de 5,8 por 100.000 mujeres el año 2010, más del doble que la de países desarrollados como Finlandia y Estados Unidos de Norteamérica (USA) ${ }^{5,6}$. La mortalidad varía ampliamente entre las distintas regiones del país; el año 2010, la región Metropolitana y de Coquimbo tuvieron tasas de 4,71 y 9,67 por 100.000 mujeres, respectivamente ${ }^{3}$. La desigualdad se extrema al comparar la mortalidad según nivel educacional, siendo 15 veces mayor en mujeres con menos de 4 años de educación que en mujeres con más de 12 años (Figura 1).

\section{Detección precoz en base a Papanicolaou}

Alrededor de 1940, George Papanicolaou demostró que en células descamativas del cuello uterino se podía identificar cambios celulares conducentes a cáncer. Desde entonces el examen de Papanicolaou ha sido la principal herramienta de prevención del CC. La efectividad de un programa de tamizaje en base a Papanicolaou depende de la cobertura, toma de muestra, entrenamiento de cito-patólogos, seguimiento de mujeres con citología positiva, oferta de diagnóstico y tratamiento

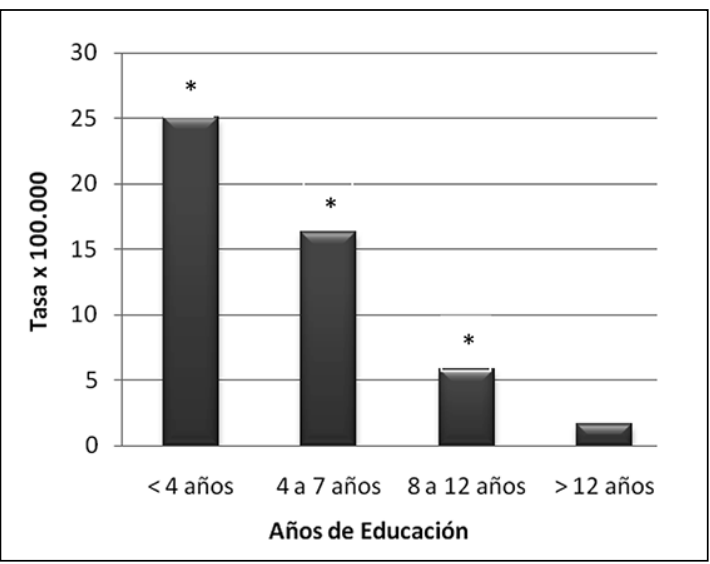

Figura 1. Tasa de mortalidad por cáncer cervicouterino ajustada por edad según años de educación para el año 2009 en Chile. ${ }^{*} p<0,001$ en relación a $>12$ años de educación. Fuente: Departamento de Estadísticas e Información de Salud. Ministerio de Salud. Gobierno de Chile. Disponible en: http:// www.deis.cl [Consultado en marzo de 2013]. oportuno ${ }^{7}$. Los programas basados en citología en USA y Finlandia han reducido importantemente la mortalidad por CC, con coberturas de $80 \%{ }^{5}$. En Chile, en 1987 se inició el tamizaje trienal con Papanicolaou en mujeres entre 25 y 64 años. La cobertura del programa se ha mantenido alrededor de $60 \%$ en los últimos años ${ }^{3}$, cobertura relativamente alta dentro de los países de la Organización para la Cooperación y el Desarrollo Económico (OCDE). Pese a esto, Chile presenta las tasas de incidencia y mortalidad por CC más altas de la OCDE después de México (Figura 2 2 $^{8}$. Esto puede estar reflejando diferencias en la calidad del Papanicolaou entre estos países ${ }^{9,10}$. La sensibilidad del Papanicolaou varía entre 30 y $87 \%$ y su especificidad entre 86 y $100 \%{ }^{11}$. En Chile, el único estudio controlado sobre sensibilidad del Papanicolaou arrojó un resultado en el límite inferior de lo reportado $(25 \%)^{12}$.

\section{Reconocimiento del VPH como causa nece- saria del CC}

El VPH se considera la infección de transmisión sexual (ITS) más frecuente en el mundo ${ }^{13}$, estimándose que más de $70 \%$ de las personas se habrán infectado alguna vez. La mayoría elimina la infección dentro de seis meses a dos años ${ }^{13}$, pero las infecciones persistentes por genotipos de $\mathrm{VPH}$ oncogénicos, conocidos como VPH de alto riesgo, causan $100 \%$ de los CC, considerándose una causa necesaria para el desarrollo de este cáncer ${ }^{14}$. En Chile, de los cánceres atribuibles a VPH, el CC representa $89,6 \%$ de los casos nuevos anuales; $4,5 \%$ de los cánceres asociados a VPH ocurren en hombres y $95,5 \%$ en mujeres ${ }^{15}$. Los estudios de prevalencia de VPH en mujeres chilenas muestran que los VPH oncogénicos infectan a $10 \%$ de las mujeres adultas ${ }^{16,17}$, se encuentran presentes en $93,6 \%$ de las lesiones precancerosas ${ }^{18}$ y en $94,2 \%$ de los cánceres invasores ${ }^{19}$. La infección por VPH ha aumentado en Chile en la última década ${ }^{20}$. Actualmente, no existe tratamiento para las infecciones por VPH; sólo se tratan las lesiones que éstas causan.

\section{Cambio de paradigma de prevención}

La identificación del VPH como causa necesaria del CC promovió el desarrollo de vacunas 


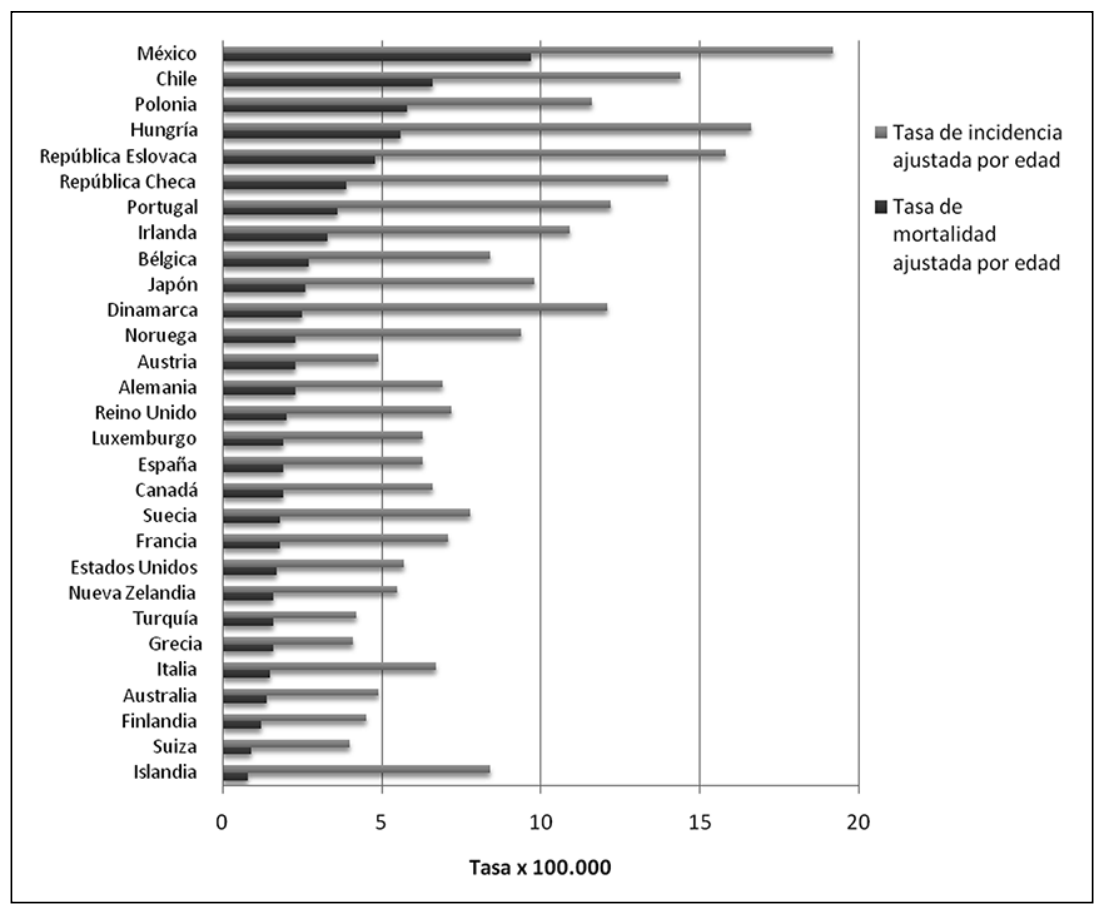

Figura 2. Tasas de incidencia y mortalidad por cáncer cervicouterino ajustadas por edad en países de la OCDE 2008. Fuente: GLOBOCAN 2008 v1.2, cancer incidence and mortality worldwide: IARC Cancer Base no. 10. Lyon, France: International agency for research on cancer; 2010. Disponible en: http:// globocan.iarc.fr. [Consultado en marzo de 2013]. para prevenir la infección y de pruebas para la detección del virus. En los últimos 20 años se ha demostrado a nivel poblacional la efectividad de estas tecnologías, las que se han ido incorporando progresivamente en los programas nacionales de prevención de países desarrollados y menos desarrollados (México, Colombia, República Dominicana, Argentina, Perú, entre otros).

\section{1. Vacunación anti-VPH}

Actualmente, hay en el mercado dos vacunas para la profilaxis de infecciones por VPH: la bivalente Cervarix ${ }^{\circledR}$ y la tetravalente Gardasil ${ }^{\circledR}$. Ambas están dirigidas contra los genotipos 16 y 18, que son responsables de cerca de $70 \%$ de los $\mathrm{CC}^{21}$; la tetravalente además agrega protección contra los genotipos 6 y 11, que se asocian a condilomas acuminados ${ }^{9}$. Estas vacunas han demostrado una protección cercana a $100 \%$ contra infecciones por los genotipos vacunales y contra lesiones intraepiteliales grado 2 o más (NIE2+) asociadas a ellos. Ambas han demostrado algún grado de eficacia para los genotipos 31, 45 y 33 entre otros, lo que sugiere la presencia de protección cruzada ${ }^{21}$. Se ha demostrado duración de la inmunogenicidad (niveles altos de IgG) a lo menos hasta 8,4 años de seguimiento de adolescentes vacunadas ${ }^{21}$. La efectividad disminuye a $53 \%$ al incluir mujeres que estuvieron expuestas al virus previo a la vacunación ${ }^{22}$, por lo que se recomienda su administración antes del inicio de la actividad sexual. El esquema de administración aprobado por los laboratorios es de tres dosis en seis meses, sin embargo, la evidencia indica que dos dosis en niñas prepúberes serían tan inmunogénicas como tres dosis en mujeres adultas ${ }^{23,24}$ y que la inmunogenicidad es mayor a mayor intervalo entre dosis ${ }^{23}$. Aunque algunos países han incluido a hombres en sus programas de vacunación, esto no es respaldado por la Organización Mundial de la Salud (OMS) por no considerarse costo-efectivo, dado que la mayor carga de enfermedad ocurre en las mujeres y ellas son el reservorio de la infección; estando ellas protegidas, se protege también a los hombres ${ }^{25}$. En países con muy bajas coberturas de vacunación en mujeres se puede considerar vacunar hombres ${ }^{21}$.

\section{Vacuna anti-VPH 16/18 en Chile}

Un estudio de costo-efectividad realizado el 2011 por el MINSAL determinó que la vacuna sería costo-efectiva a un valor inferior a US $\$ 18$ por 
dosis ${ }^{26}$; actualmente la vacuna está disponible para el programa nacional a un precio menor. En base a la evidencia disponible, el año 2012, la Comisión Nacional Asesora de Vacunas e Inmunizaciones (CAVEI) recomendó la vacunación a niñas entre 9 y 11 años $^{27}$ en dos dosis con doce meses de intervalo y, si se determinara la necesidad de una tercera dosis, se recomendó aplicarla a los 60 meses. Dada la alta cobertura de los programas de inmunización en Chile, al vacunar niñas se lograría protección de los niños por inmunidad de rebaño. Además se recomendó iniciar una vigilancia de la inmunogenicidad en las vacunadas y de la infección en la población femenina. Este programa fue aceptado y comenzaría a implementarse a partir de 2014.

\subsection{Tamizaje en base a VPH}

Actualmente, se dispone de varios exámenes de laboratorio para identificar la infección por VPH. El más usado y estudiado es la detección del ADN viral en células cervicales. Estas técnicas moleculares tienen una sensibilidad mayor de $95 \%$ para detectar NIE $2+{ }^{28}$. En un estudio poblacional en India que comparó distintas alternativas de tamizaje, un solo examen de VPH disminuyó en 50\% la incidencia y mortalidad por CC, mientras que un solo examen de Papanicolaou no tuvo efecto ${ }^{29}$. Otro beneficio del tamizaje basado en VPH es que la muestra puede ser recolectada por la misma mujer. Los resultados obtenidos por autotoma tienen una correlación con los obtenidos de muestras de células cervicales mayor a $90 \%{ }^{30}$. La autotoma para detección de VPH se ha utilizado en países latinoamericanos como México ${ }^{31}$, Perú $^{32}$, Brasil $^{33}$ y Chile ${ }^{34}$, demostrando una buena aplicabilidad y una alta aceptabilidad ${ }^{35}$. En Chile, la autotoma fue utilizada exitosamente en la encuesta nacional de salud de $2003^{17}$ y más recientemente en un estudio de mujeres no-adherentes a tamizaje con Papanicolaou de la Región Metropolitana ${ }^{34}$, de la cuales $86,5 \%$ aceptó realizarse autotoma. Esta estrategia podría ser utilizada para rescatar a mujeres que no cumplen con el tamizaje, aumentando la cobertura de un programa nacional de prevención del CC, situación que ha sido demostrada en México ${ }^{36}$.

Desde el punto de vista de su implementación y control de calidad, los exámenes de VPH tienen como ventaja sobre el Papanicolaou que son técnicas automatizadas fácilmente estandarizables y requieren menos personal. Recientemente la
OMS publicó una nueva guía para la prevención del $\mathrm{CC}^{37}$, en la cual por primera vez se recomienda la utilización de la prueba de VPH en aquellos lugares que no tienen un programa o tienen uno en base a inspección visual con ácido acético (IVA) y en aquellos lugares que tienen un programa basado en citología pero que no cumple con criterios de calidad; en los casos en que el programa sí cumple con criterios de calidad, la indicación es usar cualquiera de las dos opciones, citología o prueba de VPH. El principal problema de la detección de VPH para identificar lesiones pre-cancerosas es su alta tasa de falsos positivos, porque las infecciones por VPH de alto riesgo son muy frecuentes, especialmente en mujeres jóvenes $(22,4 \%$ en mujeres menores de 25 años en Chile $)^{17}$, y la mayoría de ellas son transitorias y no están asociadas a lesiones. Por esta razón, no se recomienda el tamizaje primario con VPH en mujeres menores de 30 años. Por otra parte, derivar a colposcopía a todas las mujeres VPHpositivas representaría una sobrecarga innecesaria al sistema de salud y a la mujer. Por ello, se están evaluando diversas estrategias para seleccionar a las mujeres portadoras de VPH que requieren derivación, proceso conocido como triage.

\section{VPH en prevención secundaria en Chile}

El uso de la detección de VPH como tamizaje primario fue evaluado en un ensayo de campo que reclutó 8.407 mujeres entre 25 y 64 años de edad residentes de la comuna de Puente Alto, quienes recibieron una prueba de VPH y un Papanicolaou simultáneamente ${ }^{12}$. De las mujeres diagnosticadas con una lesión NIE3+, 57,4\% sólo resultaron positivas a la detección de VPH y no habrían sido detectadas a través del Papanicolaou. El Papanicolaou detectó sólo 55,5\% de los CC encontrados en el estudio a partir de la detección de VPH (Tabla 1). La sensibilidad del Papanicolaou para detectar lesiones NIE3+ en este estudio fue de 23,6\% (IC $95 \% 15,1-34,9$ ) comparado con $94,9 \%$ (IC 95\% $82,5-98,7)$ de la prueba de VPH. La especificidad del VPH para lesiones NIE3+ fue menor que la del Papanicolaou, 91,0\% (IC 95\% 90,6-91,5) y 98,7\% (IC 95\% 98,5-98,8) respectivamente, y sólo $10,5 \%$ de las mujeres VPH-positivas presentaron una lesión precancerosa (NIE2+) comparado con 23,9\% de las Papanicolaou-positivas. La tasa de derivación al nivel secundario aumentó de 2 a $10 \%$ con el uso de la prueba de VPH. 
Tabla 1. Resultados del tamizaje de 8.407 mujeres chilenas con examen de VPH y Papanicolaou

\begin{tabular}{|lcccc|} 
& VPH (+) Pap (-) & VPH (-) Pap (+) & VPH (+) Pap (+) & VPH (-) Pap (-)* \\
$\mathbf{n = 7 9 3}$ & $\mathbf{n}=\mathbf{4 8}$ & $\mathbf{n = 9 0}$ & $\mathbf{2 9 5}$ \\
NIE2 & 27 & 0 & 12 & 3 \\
NIE3 & 28 & 0 & 16 & 1 \\
Cáncer & 3 & 0 & 5 & 1 \\
\hline
\end{tabular}

*Grupo control de alto riesgo: mujeres negativas a VPH y a Pap, con IVA positiva o factores de riesgo para CC. Adaptado de Ferreccio et al, Int J Cancer, 2013 (12).

\subsection{Triage de un examen VPH positivo}

Entre las opciones de triage se consideran el examen visual (sea colposcópico o mediante IVA), la citología convencional o de base líquida, y la utilización de técnicas moleculares como la genotipificación de los tipos 16 y 18 , la identificación de proteínas virales y la detección de ARNm de VPH de alto riego, entre otros. En Holanda, el seguimiento con Papanicolaou (sensibilidad del Papanicolaou 70\% $)^{38}$ alcanzó el mayor valor predictivo positivo (VPP), con la menor tasa de derivación a colposcopía, pero una menor sensibilidad, en comparación con distintas estrategias de genotipificación del virus ${ }^{39}$. En Canadá, la detección de VPH seguido por triage con Papanicolaou (sensibilidad del Papanicolaou de 59\%) sería más costo-efectiva en reducir años de vida perdidos ajustados por discapacidad (QALYs) que el Papanicolaou anual ${ }^{40}$. En el estudio ATHENA en USA, la genotipificación VPH16 y VPH18 fue equivalente a la citología en términos de sensibilidad y VPP. Las mejores alternativas fueron aquellas que incluyeron ambas estrategias ${ }^{41}$. Menos estudiado es el rol de la colposcopía como triage. En distintas partes del mundo desarrollado se ha incorporado la colposcopía tanto diagnóstica como terapéutica como parte de las habilidades del médico familiar. En un estudio australiano, los médicos familiares fueron capacitados en la realización de colposcopías alcanzando un nivel de competencia equivalente al del especialista ${ }^{42}$. En Estados Unidos de Norteamérica existen también buenas experiencias de entrenamiento y práctica de colposcopía por médicos y enfermeras de atención primaria de salud (APS) ${ }^{43,44}$, así como experiencias efectivas con el uso de cono ${ }^{45}$ y la escisión electroquirúrgica con asa $(\text { LEEP })^{46}$, logrando una alta tasa de márgenes negativos, tasas de recurrencia y nivel de complicaciones similares a las del especialista. En Chile existen experiencias exitosas en la capacitación de médicos familiares en procedimientos médico-quirúrgicos como endoscopía digestiva alta y endosonografía abdominal, así como en entrenamiento en IVA para matronas.

\section{Propuesta de incorporación del examen de VPH en Chile}

En base a lo revisado, la Figura 3 resume una propuesta para la incorporación de la detección de VPH al programa nacional de prevención de CC en Chile. El tamizaje primario se realizaría con una prueba de VPH. Las mujeres con resultado negativo continuarían tamizaje rutinario en intervalos de 3 a 5 años. Las mujeres VPH-positivas recibirían una de las siguientes opciones de triage: a) Colposcopía de triage; ésta sería realizada en APS por un profesional capacitado en la técnica y se distingue de una colposcopía realizada por un especialista en que el objetivo es sólo diferenciar un cuello normal de uno anormal; b) Prueba de triage refleja; ésta se realizaría en la misma muestra tomada para la prueba de VPH y puede ser una prueba molecular o citología de base líquida.

Si el resultado del triage es negativo, la mujer se controlaría en 1 a 3 años. Si el triage es positivo, la mujer se derivaría al ginecólogo para colposcopía diagnóstica y continuar el manejo según la norma ministerial vigente.

La Sociedad Americana del Cáncer y el Colegio Americano de Ginecobstetras recomiendan el cotamizaje con Papanicolaou y detección de VPH en mujeres entre 30 y 65 años $^{47}$. Esta estrategia de co-tamizaje, si bien incrementa los costos, podría considerarse en Chile en una fase inicial, para familiarizar a los equipos de salud con el nuevo examen. 


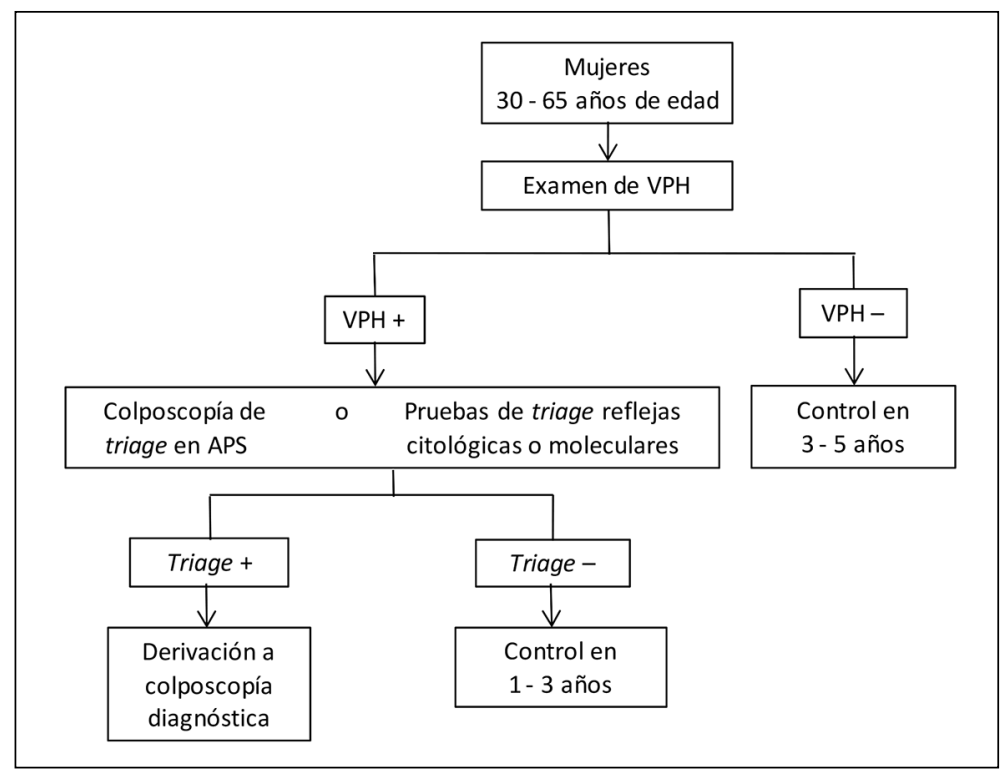

Figura 3. Propuesta para la implementación de detección de VPH como parte del programa de prevención de cáncer cervicouterino en Chile.

\section{Conclusiones}

La incorporación de estrategias de prevención primaria y secundaria basadas en VPH podría contribuir a disminuir la inequidad existente en Chile con respecto al CC. El programa actual basado en tamizaje mediante Papanicolaou, incluso con los mayores esfuerzos, no logra la cobertura óptima de $80 \%$, lo que se ve agravado por la baja sensibilidad de esta técnica. Usar una estrategia de tamizaje con mayor sensibilidad, que permita un mayor intervalo entre exámenes y que tenga la opción de autoexamen, como es la detección de VPH, permitiría en corto plazo disminuir la brecha en la mortalidad. Las mujeres de niveles socioeconómicos bajos serían las más beneficiadas con un tamizaje más sensible y menos frecuente.

Utilizar la prueba de VPH debe considerar algunos aspectos: la infección por $\mathrm{VPH}$, al corresponder a una ITS, puede generar importantes consecuencias emocionales y sociales. Para minimizar las reacciones adversas, este examen debe acompañarse de educación sobre la historia natural de la enfermedad y la alta prevalencia de la infección en la población. Por otro lado, se deben considerar los riesgos inherentes a un tamizaje altamente sensible, como son el sobrediagnóstico y sobre-tratamiento, que conllevan costos personales y sociales. Para que el balance de incorporar la prueba de VPH resulte a favor de los beneficios, se debe considerar un proceso de triage para las mujeres VPH-positivas de modo de aumentar el VPP del examen. Aún no se dispone de normas internacionales respecto a métodos de triage, habiendo varios estudios en curso. El avance de la investigación científica entregará luces sobre qué tipo de triage será el más adecuado a nuestro contexto.

En un escenario en que la vacunación antiVPH esté incorporada universalmente en el país y las generaciones vacunadas hayan alcanzado la edad de tamizaje, el uso de técnicas basadas en VPH será una necesidad indiscutible. Por un lado, el número de mujeres con lesiones precancerosas será mucho menor, por lo que el tamizaje con citología implicaría una gran proporción de citologías negativas, pudiendo llevar a un deterioro del desempeño de este examen debido a su naturaleza altamente subjetiva y repetitiva. Por otro lado, las lesiones que se detectarían citológicamente serán en su gran mayoría causadas por agentes menos oncogénicos y que no progresarán ${ }^{47,48}$.

La larga experiencia de los equipos de APS en la implementación de un programa como el actualmente existente, así como la cultura preventiva de esta enfermedad en nuestra población, son recursos sociales que aportan a la posibilidad de un cambio exitoso en nuestro país. Dentro de los 
desafíos en la implementación de una nueva política de prevención, está el problema de los recursos frescos que se deben movilizar para incorporar un nuevo test de tamizaje. Asimismo, modificar las actividades del proceso clínico y administrativo en la toma del Papanicolaou a lo largo del país puede ser resistido por temor a perder lo avanzado. Actualizar a prestadores y a la población respecto a esta enfermedad son aspectos claves para lograr una modificación del programa.

Agradecimientos. Agradecemos al doctor Jorge Brañes y a su equipo de ginecología, a la biólogo molecular Helena Poggi, a la epidemióloga Solana Terrazas, a la estadístico Francisca González y a todo el equipo del proyecto de investigación FONDECYT No 1090597 a partir del cual se han desarrollado las ideas de este artículo.

\section{Referencias}

1. Drain PK, Holmes KK, Hughes JP, Koutsky LA. Determinants of cervical cancer rates in developing countries. Int J Cancer 2002; 100 (2): 199-205.

2. IARC Working Group on the Evaluation of Carcinogenic Risks to Humans. Human papillomaviruses. IARC Monogr Eval Carcinog Risks Hum 1995; 64: 1-378.

3. Departamento de Estadísticas e Información en Salud. Disponible en: http://www.deis.minsal.cl [Consultado en diciembre de 2012].

4. Objetivos sanitarios de la década 2000-2010. Evaluación final del período. Objetivos de impacto. Ministerio de salud, Gobierno de Chile. Disponible en: http://www. minsal.gob.cl/portal/url/item/94c89f56c4e270b0e0400 1011e011c9c.pdf. [Consultado en marzo de 2013].

5. van der Aa MA, Pukkala E, Coebergh JW, Anttila A, Siesling S. Mass screening programmes and trends in cervical cancer in Finland and the Netherlands. Int J Cancer 2008a; 122 (8): 1854-8.

6. SEER cancer statistics review, 1975-2006, National Cancer Institute. Disponible en: http://seer.cancer.gov/ csr/1975_2006. [Consultado en marzo de 2013].

7. Murillo R, Almonte M, Pereira A, Ferrer E, Gamboa OA, Jeronimo J, et al. Cervical cancer screening programs in latin america and the caribbean. Vaccine 2008a; 26 Suppl 11: L37-48.

8. Organisation for Economic Co-operation and Development, Health at a Glance 2011: OECD Indicators, OECD Publishing.

9. Almonte M, Murillo R, Sánchez GI, Jeronimo J, Salme- ron J, Ferreccio C, et al. New paradigms and challenges in cervical cancer prevention and control in Latin America. Salud Pública Mex 2010; 52 (6): 544-59.

10. DeMay RM. Cytopathology of false negatives preceding cervical carcinoma. Am J Obstet Gynecol 1996; 175 (4 Pt 2): 1110-3.

11. Nanda K, McCrory DC, Myers ER, Bastian LA, Hasselblad V, Hickey JD, et al. Accuracy of the papanicolaou test in screening for and follow-up of cervical cytologic abnormalities: A systematic review. Ann Intern Med 2000; 132 (10): 810-9.

12. Ferreccio C, Barriga MI, Lagos M, Ibáñez C, Poggi H, González F, et al. Screening trial of human papillomavirus for early detection of cervical cancer in Santiago, Chile. Int J Cancer 2013; 132 (4): 916-23.

13. Wheeler CM. The natural history of cervical human papillomavirus infections and cervical cancer: Gaps in knowledge and future horizons. Obstet Gynecol Clin North Am 2013; 40 (2): 165-76.

14. de Martel C, Ferlay J, Franceschi S, Vignat J, Bray F, Forman D, et al. Global burden of cancers attributable to infections in 2008: A review and synthetic analysis. Lancet Oncol 2012; 13 (6): 607-15.

15. Departamento de Epidemiología. Primer informe de registros poblacionales de cáncer de Chile. Quinquenio 2003-2007. Unidad de Vigilancia de Enfermedades No transmisibles y Estudios. Departamento de Epidemiología. Ministerio de Salud. Chile; 2012. Disponible en: http://epi.minsal.cl/epi/0notransmisibles/cancer/ INFORME\%20RPC\%20CHILE\% 202003-2007,\%20 UNIDAD\%20VENT,\%20DEPTO.EPIDEMIOLOGIAMINSAL,13.04.2012.pdf [Consultado en junio de 2013].

16. Ferreccio C, Corvalán A, Margozzini P, Viviani P, González C, Aguilera X, et al. Baseline assessment of prevalence and geographical distribution of HPV types in Chile using self-collected vaginal samples. BMC Public Health. 2008; 8: 78.

17. Ferreccio C, Prado RB, Luzoro AV, Ampuero SL, Snijders PJ, Meijer CJ, et al. Population-based prevalence and age distribution of human papillomavirus among women in Santiago, Chile. Cancer Epidemiol Biomarkers Prev 2004; 13 (12): 2271-6.

18. Ili CG, Brebi P, López J, García P, Leal P, Suárez E, et al. Genotyping of human papillomavirus in cervical intraepithelial neoplasia in a high-risk population. J Med Virol 2011; 83 (5): 833-7.

19. Roa JC, García P, Gómez J, Fernández W, Gaete F, Espinoza A, et al. HPV genotyping from invasive cervical cancer in Chile. Int J Gynaecol Obstet 2009; 105 (2): 150-3.

20. Ferreccio C, Van De Wyngard V, Olcay F, Domínguez 
MA, Puschel K, Corvalán AH, et al. High-risk HPV infection after five years in a poputation-based cohort of Chilean women. Infect Agent Cancer 2011; 6 (1): 21.

21. Lehtinen M, Dillner J. Clinical trials of human papillomavirus vaccines and beyond. Nat Rev Clin Oncol 2013; 10 (7): 400-10.

22. Romanowski B. Long term protection against cervical infection with the human papillomavirus: Review of currently available vaccines. Hum Vaccin 2011; 7 (2): 161-9.

23. Romanowski B, Schwarz TF, Ferguson LM, Peters K, Dionne M, Schulze K, et al. Immunogenicity and safety of the HPV-16/18 AS04-adjuvanted vaccine administered as a 2-dose schedule compared with the licensed 3-dose schedule: Results from a randomized study. Hum Vaccin 2011; 7 (12): 1374-86.

24. Dobson SR, McNeil S, Dionne M, Dawar M, Ogilvie G, Krajden M, et al. Immunogenicity of 2 doses of HPV vaccine in younger adolescents vs 3 doses in young women: A randomized clinical trial. JAMA 2013; 309 (17): 1793-802.

25. Human papillomavirus vaccines. WHO position paper. Wkly Epidemiol Rec 2009; 84 (15): 118-31.

26. Castillo M, Castillo C. La costo-efectividad potencial de una vacuna contra el VPH en Chile. Boletín de Economía y Salud 2011; 5: 2-12.

27. Ministerio de Salud, Gobierno de Chile. Programa nacional de inmunizaciones. Disponible en: http://web. minsal.cl/sites/default/files/files/VPHCAVEIRecomendacionOctubre2012.pdf. [Consultado en abril de 2014].

28. Szarewski A, Ambroisine L, Cadman L, Austin J, Ho L, Terry G, et al. Comparison of predictors for high-grade cervical intraepithelial neoplasia in women with abnormal smears. Cancer Epidemiol Biomarkers Prev 2008; 17 (11): 3033-42.

29. Sankaranarayanan R, Nene BM, Shastri SS, Jayant K, Muwonge R, Budukh AM, et al. HPV screening for cervical cancer in rural India. N Engl J Med 2009; 360 (14): 1385-94.

30. Petignat P, Faltin DL, Bruchim I, Tramer MR, Franco EL, Coutlee F. Are self-collected samples comparable to physician-collected cervical specimens for human papillomavirus DNA testing? A systematic review and meta-analysis. Gynecol Oncol 2007; 105 (2): 530-5.

31. Salmeron J, Lazcano-Ponce E, Lorincz A, Hernández M, Hernández P, Leyva A, et al. Comparison of HPV-based assays with papanicolaou smears for cervical cancer screening in Morelos state, Mexico. Cancer Causes Control 2003; 14 (6): 505-12.

32. Baldwin S, Santos C, Méndez Brown E, Nuno T, Giuliano A, Davis J, et al. Comparison of type-specific human papillomavirus data from self and clinician directed sampling. Gynecol Oncol 2005; 97 (2): 612-7.

33. Longatto-Filho A, Roteli-Martins $\mathrm{C}$, Hammes L, Etlinger D, Pereira SM, Erzen M, et al. Self-sampling for human papillomavirus (HPV) testing as cervical cancer screening option. Experience from the LAMS study. Eur J Gynaecol Oncol 2008; 29 (4): 327-32.

34. Leniz J, Barriga M, Lagos M, Ibáñez C, Puschel K, Ferreccio C. Autotoma vaginal para detección de virus papilloma humano en mujeres no adherentes a papanicolaou en Chile. Salud Pública Mex 2013; 55: 162-9.

35. Huynh J, Howard M, Lytwyn A. Self-collection for vaginal human papillomavirus testing: Systematic review of studies asking women their perceptions. J Low Genit Tract Dis 2010; 14 (4): 356-62.

36. Lazcano-Ponce E, Lorincz AT, Cruz-Valdez A, Salmerón J, Uribe P, Velasco-Mondragón E, et al. Self-collection of vaginal specimens for human papillomavirus testing in cervical cancer prevention (MARCH): A communitybased randomised controlled trial. The Lancet; 378 (9806): 1868-73.

37. World Health Organization 2013. WHO guidelines for screening and treatment of precancerous lesions for cervical cancer prevention. WHO Press, World Health Organization, 20 Avenue Appia, 1211 Geneva 27, Switzerland.

38. de Kok IM, van Rosmalen J, Dillner J, Arbyn M, Sasieni $\mathrm{P}$, Iftner T, et al. Primary screening for human papillomavirus compared with cytology screening for cervical cancer in european settings: Cost effectiveness analysis based on a dutch microsimulation model. BMJ 2012; 344: e670.

39. Rijkaart DC, Berkhof J, van Kemenade FJ, Coupe VM, Hesselink AT, Rozendaal L, et al. Evaluation of 14 triage strategies for HPV DNA-positive women in populationbased cervical screening. Int J Cancer 2012; 130 (3): 602-10.

40. Vijayaraghavan A, Efrusy MB, Mayrand MH, Santas CC, Goggin P. Cost-effectiveness of high-risk human papillomavirus testing for cervical cancer screening in Quebec, Canada. Can J Public Health 2010; 101 (3): 220-5.

41. Castle PE, Stoler MH, Wright Jr TC, Sharma A, Wright TL, Behrens CM. Performance of carcinogenic human papillomavirus (HPV) testing and HPV16 or HPV18 genotyping for cervical cancer screening of women aged 25 years and older: A subanalysis of the ATHENA study. The Lancet Oncology 2011; 12 (9): 880-90.

42. Cherry S, Blackledge D, Russell R. Colposcopy in general practice. Aust Fam Physician. 1996; 25 (11): 1737-42.

43. Ferris DG, Miller MD. Colposcopy practice and training 
in family practice residency programs. J Am Board Fam Pract 1992; 5 (2): 153-6.

44. Phillips WR. Diagnostic and screening procedures in family practice. Past, present, and future use. Arch Fam Med 1993; 2 (10): 1051-7.

45. Mulhem E, Kennedy EL, Lick D. Treatment of cervical dysplasia with the fischer cone biopsy excisor in a family medicine office: A case series. J Am Board Fam Med 2010; 23 (2): 154-8.

46 Lyman DJ, Morris B. LEEP in the family practice setting.
J Am Board Fam Pract 2003; 16 (3): 204-8.

47. Franco E, Tsu V, Herrero R, Lazcano-Ponce E, Hildesheim A, Muñoz N, et al. Integration of Human Papillomavirus Vaccination and Cervical Cancer Screening in Latin America and the Caribbean. Vaccine 2008; 26S: L88-L95.

48. Franco E, Cuzick J, Hildesheim A, de Sanjosé S. Chapter 20: Issues in planning cervical cáncer screening in the era of HPV vaccination. Vaccine 2006; 24 (S3): S171S177. 